\title{
Bibnum
}

Textes fondateurs de la science

Mathématiques | 2017

\section{Liouville, pionnier des développements en séries de fonctions orthogonales}

Alain Juhel

\section{(2) OpenEdition}

Journals

Édition électronique

URL : https://journals.openedition.org/bibnum/599

DOI : 10.4000/bibnum.599

ISSN : 2554-4470

\section{Éditeur}

FMSH - Fondation Maison des sciences de l'homme

Référence électronique

Alain Juhel, «Liouville, pionnier des développements en séries de fonctions orthogonales », Bibnum [En ligne], Mathématiques, mis en ligne le 01 juin 2010, consulté le 04 février 2023. URL : http:// journals.openedition.org/bibnum/599; DOI : https://doi.org/10.4000/bibnum.599 


\section{Liouville, pionnier des développements en séries de fonctions orthogonales}

Alain Juhel, professeur de mathématiques spéciales (MP) au lycée Faidherbe, Lille

En 1836, Joseph Liouville, formé à l'École polytechnique, puis à celle des Ponts \& chaussées, est âgé de 27 ans, et il vient de fonder le Journal de mathématiques pures \& appliquées, qui prend le relais des Annales de Gergonne, disparues en 1832. Brillant animateur, disposant d'un réseau de collègues dont il sollicite les publications inédites (une cinquantaine d'auteurs différents y écrivent dès les cinq premières années), il n'hésite pas à mettre lui-même, souvent copieusement, la main à la pâte : c'est ainsi que le tout premier numéro contient son « Mémoire sur le développement des fonctions ou parties de fonctions dont les divers termes sont assujettis à satisfaire une même équation différentielle du second ordre contenant un paramètre variable », premier d'une série de trois dont les deux suivants paraîtront en 1837. Quelques pages plus loin, un mémoire de son ami Charles Sturm (1803-1855), « Sur les équations différentielles linéaires du second ordre », que celui de Liouville prolonge. Les deux articles, échos de présentations légèrement antérieures à l'Académie, jettent ainsi les bases de ce que l'on appellera désormais Théorie de Sturm-Liouville.

Le premier mémoire de Liouville qu'on se propose de présenter ici est important à trois titres :

1. Il généralise de façon spectaculaire l'analyse de Fourier qui en a inspiré la genèse ;

2. Il contribue, par des lacunes inévitables pour l'époque, à ouvrir de nouvelles orientations en mathématiques au tournant du $X X^{\mathrm{e}}$ siècle, avec l'étude, par Hilbert notamment, de nouveaux modes de convergence, de nouveaux espaces de fonctions ;

3. Il donne à la physique des outils essentiels, considérés aujourd'hui comme 
indispensables, de l'étude des vibrations mécaniques ou électromagnétiques jusqu'à la mécanique quantique.

Dès son premier numéro, le Journal de Liouville pouvait difficilement mieux justifier les deux qualificatifs de son titre : pures \& appliquées. Un seul thème, décliné en deux angles d'attaque différents, chacun propre à son auteur, allait montrer dans son développement futur l'arbitraire de ces commodités de classification (et, parfois, sources de conflits universitaires...).

\section{LE PROBLEME DE STURM- LI OUVI LLE PAR L'EXEM PLE}

Afin de mieux suivre le texte et les notations de Liouville, commençons par étudier quelques exemples explicites. Ce faisant, nous nous mettons dans les pas de Fourier et de sa théorie de la chaleur $(1807,1811)$, une filiation pleinement revendiquée par Liouville dès sa première phrase :

Lorsqu'on veut déterminer les lois du mouvement de la chaleur dans une barre hétérogène (...)

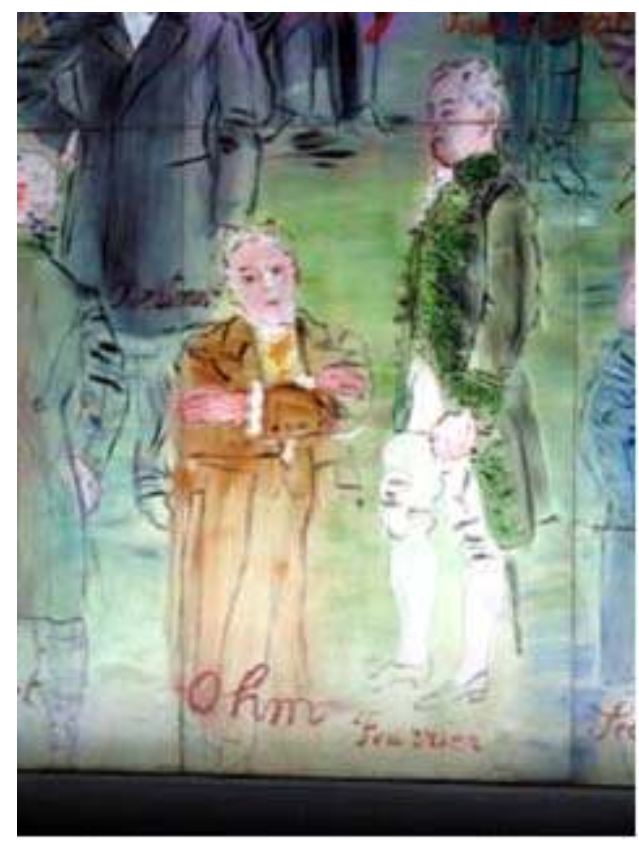

Figure 1 : à droite, Joseph Fourrier [sic], en habit d'académicien. Joseph Fourier (1768 - 1830) a été Secrétaire perpétuel de l'Académie des sciences de 1821 jusqu'à sa mort en 1830. Détail de La Fée Electricité, Raoul Dufy (Palais de Chaillot, 1937). Photographie Alain Juhel. 
Exemple 1: barre homogène, même température fixe aux extrémités.

La température en un point d'abscisse $x$, au temps $t$, dans une barre homogène de longueur $I^{1}$, est régie par les équations (1), (2) et (3) :

$$
\begin{aligned}
& \text { (1) } \frac{\partial \mathrm{u}}{\partial \mathrm{t}}=\frac{\partial^{2} \mathrm{u}}{\partial \mathrm{x}^{2}} \\
& \text { (2) } \mathrm{u}(0, \mathrm{t})=0 ; \mathrm{u}(\mathrm{l}, \mathrm{t})=0 \\
& \text { (3) } \mathrm{u}(\mathrm{x}, 0)=\mathrm{f}(\mathrm{x})
\end{aligned}
$$

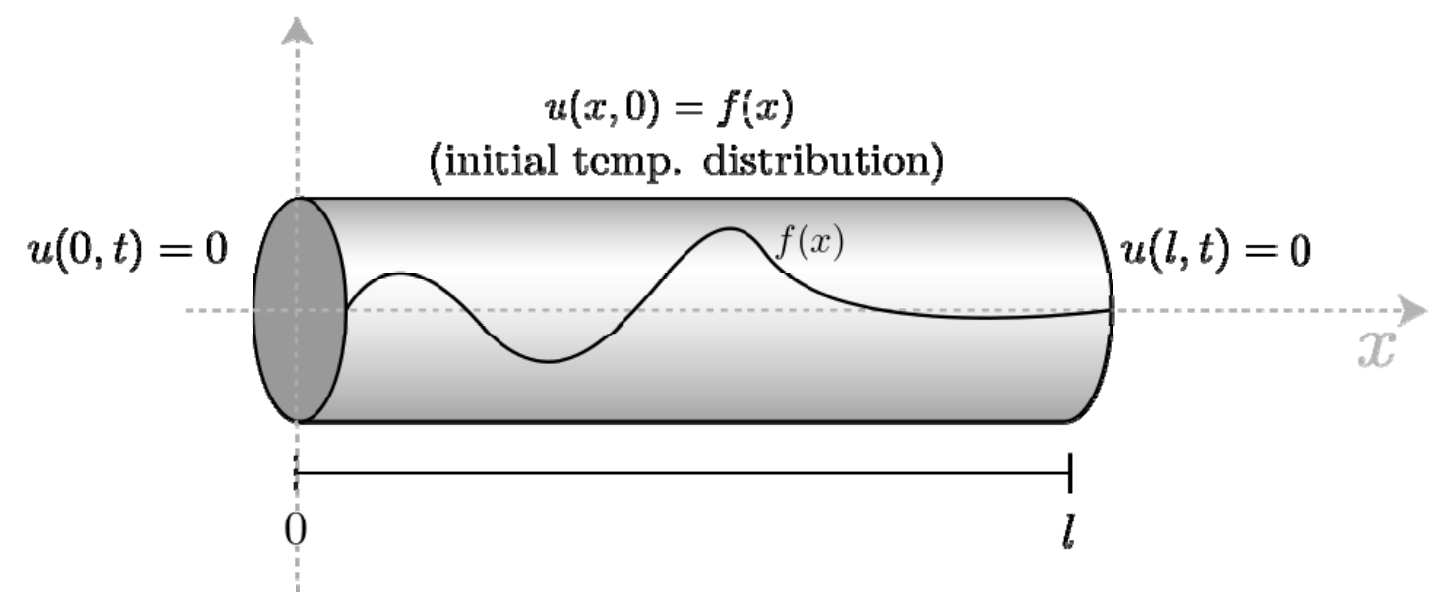

La relation (1) représente la loi d'évolution de la température dans la barre en fonction des deux variables naturelles : c'est une équation aux dérivées partielles. (2) fixe ce que l'on appelle les conditions aux limites (ici, les abscisses 0 et $/$ ). (3) fixe la donnée initiale, c'est à dire la répartition initiale des températures dans la barre: dans une de ses toutes premières expériences, Fourier l'avait uniformément chauffée $(f(x)=$ C) avant d'installer ses extrémités dans la glace (2), et de laisser la barre se refroidir selon (1). Intuitivement, le problème semble «physiquement » bien déterminé, et l'on n'imagine guère l'expérience fournir des résultats différents si on la répète, ce que Fourier eut tout le loisir de constater. Mathématiquement, c'est dire que le problème admet une solution unique : c'est vrai, mais il n'est pas immédiat de faire la démonstration qui corrobore notre intuition physique.

1. Nous commençons, comme Fourier, par ce cas où l'équation est bien plus simple. Par ailleurs, pour des raisons de graphie comme de simplification, on prendra $I=1$ par la suite. 
Il est le plus souvent impossible, sauf dans des cas très simples, d'obtenir sous forme explicite les solutions d'équations telles que (1). Pour contourner cette difficulté, Fourier a eu, dès 1807, l'idée de séparer les variables, c'est à dire chercher des solutions de (1) sous la forme

$$
\mathrm{u}(\mathrm{x}, \mathrm{t})=\mathrm{v}(\mathrm{x}) \cdot \mathrm{W}(\mathrm{t})
$$

Ce faisant, il risque d'en laisser échapper beaucoup... temporairement ! Car cette forme choisie est tout ce qu'il y a de plus arbitraire. Elle n'est dictée que par la possibilité de résoudre des équations différentielles portant sur des fonctions d'une seule variable : le problème est plus simple (ou moins difficile) à traiter. Et de fait, (1) devient

$$
\mathrm{V}(\mathrm{x}) \cdot \mathrm{W}^{\prime}(\mathrm{t})=\mathrm{V}^{\prime \prime}(\mathrm{x}) \cdot \mathrm{W}(\mathrm{t})
$$

On choisit alors un $x_{0}$ et un $t_{0}$ tels que $\mathrm{V}\left(\mathrm{x}_{0}\right) \cdot \mathrm{W}\left(\mathrm{t}_{0}\right) \neq 0$. Fixant $t=t_{0}$, on constate que $V$ vérifie une équation du type

$$
\text { (A) } \quad V^{\prime \prime}(x)=-r . V(x)
$$

et de même, fixant $x=x_{0}$, que $W$ vérifie une équation du type

$$
\text { (A') } W^{\prime}(t)=-s . W(t)
$$

où $r$ et $s$ sont des réels (le signe - est une commodité). Enfin, prenant simultanément $x=x_{0}$ et $t=t_{0}$, on constate que $r=s$. On vérifie immédiatement, en retour, que ces produits fournissent des solutions de (1) ; mais on a totalement laissé de côté, pour l'instant, les conditions (2) et (3).

Pour ces solutions, vérifier (2) - pour tout $t$ - est acquis dès que la fonction $V$ est telle que :

$$
\text { (B) } \mathrm{V}(0)=0 ; \mathrm{V}(1)=0
$$

Il n'y a plus qu'à regarder si cela est possible, selon le signe de $r$ et la forme des solutions associées :

\begin{tabular}{|c|c|c|}
\hline Signe de $r$ & Solutions de (A) & Solutions de (A) et (B) \\
\hline$r=-\omega^{2}<0$ & $\mathrm{~V}(\mathrm{x})=\mathrm{a} \cdot \mathrm{e}^{\omega \mathrm{x}}+\mathrm{b} \cdot \mathrm{e}^{-\omega \mathrm{x}}$ & 0 \\
\hline$r=0$ & $\mathrm{~V}(\mathrm{x})=\mathrm{ax}+\mathrm{b}$ & 0 \\
\hline$r=\omega^{2}<0$ & $\mathrm{~V}(\mathrm{x})=\mathrm{a} \cdot \cos (\omega \mathrm{x})+\mathrm{b} \cdot \sin (\omega \mathrm{x})$ & $\mathrm{b} \cdot \sin (\pi \mathrm{nx})$ avec $r=(\pi \mathrm{n})^{2}$ \\
\hline
\end{tabular}


En résumé, il n'y a de solutions non triviales que pour une famille exceptionnelle, dénombrable, de valeurs de $r$; ce sont les solutions de l'équation transcendante - mais néanmoins facile ! - obtenue en $x=1$

$$
\text { (C) } \sin (\sqrt{r})=0
$$

cependant que l'autre condition, en $x=0$, a éliminé tout terme en cosinus.

L'équation (1) étant linéaire, toute combinaison linaire de solutions est encore solution ; cela permet d'enrichir l'ensemble des solutions de (1) et (2) en construisant des fonctions qui ne sont pas elles-mêmes des produits séparés, mais de la forme (sachant que $W(t)$ peut être mis sous la forme $e^{-s t}$ )

$$
\mathrm{u}(\mathrm{x}, \mathrm{t})=\sum_{\mathrm{n}=1}^{\mathrm{N}} \beta_{\mathrm{n}} \mathrm{V}_{\mathrm{n}}(\mathrm{x}) \mathrm{W}_{\mathrm{n}}(\mathrm{t})=\sum_{\mathrm{n}=1}^{\mathrm{N}} \beta_{\mathrm{n}} \mathrm{e}^{-(\pi \mathrm{n})^{2} \mathrm{t}} \sin (\pi \mathrm{nx})
$$

et, si l'on est un peu plus audacieux - Fourier l'était, tout en gardant un remarquable contrôle sur sa vitesse (si l'on ose dire) grâce au sens physique du problème

$$
\mathrm{u}(\mathrm{x}, \mathrm{t})=\sum_{\mathrm{n}=1}^{\infty} \beta_{\mathrm{n}} \mathrm{V}_{\mathrm{n}}(\mathrm{x}) \mathrm{W}_{\mathrm{n}}(\mathrm{t})=\sum_{\mathrm{n}=1}^{\infty} \beta_{\mathrm{n}} \mathrm{e}^{-(\pi \mathrm{n})^{2} \mathrm{t}} \sin (\pi \mathrm{nx})
$$

Une telle écriture en série (définie comme limite des sommes prises jusqu'à $N$ ) exige évidemment qu'on en étudie sa convergence, mais Fourier ne s'en était pas occupé, estimant que la forte décroissance des exponentielles l'assurerait quels que soient les coefficients $\beta_{n}$.

Reste à ajuster ces coefficients, mais c'est le moment de se souvenir que la condition (3) attend toujours son entrée en scène: nous y reviendrons un peu plus loin.

\section{@@@@@@@}

Exemple 2: une légère modification à une extrémité.

Supposons que nous considérions le même problème, modifiant seulement (2), et plus précisément la condition à la limite 1 en

$$
\text { (2) } \mathrm{u}(0, \mathrm{t})=0 ; \frac{\partial \mathrm{u}}{\partial \mathrm{x}}(1, \mathrm{t})-\mathrm{u}(1, \mathrm{t})=0
$$

sans nous arrêter à son interprétation physique. Le traitement du problème est 
similaire, mais (B) est remplacée par

(B) $\mathrm{V}(0)=0 ; \mathrm{V}^{\prime}(1)-\mathrm{V}(1)=0$

et la famille des solutions de (A) et (B) par la famille des fonctions $V_{n}(x)=\sin \left(\omega_{n} x\right)$ avec des $\omega_{n}$ donnés par l'étude de l'équation

$$
\text { (C) } \tan (\sqrt{r})=(\sqrt{r})
$$

Une étude graphique convaincra, dans un premier temps, de l'existence d'une infinité dénombrable de racines ; il sera d'ailleurs facile de le démontrer par l'étude de la fonction auxiliaire $\tan (x)-x$.

@@@@@@@

Exemple 3 : cylindre homogène, température fixée au bord.

L'équation (1) est, dans sa forme la plus générale

$$
\frac{\partial u}{\partial t}=\Delta u=\frac{\partial^{2} u}{\partial x^{2}}+\frac{\partial^{2} u}{\partial y^{2}}+\frac{\partial^{2} u}{\partial z^{2}}
$$

L'opérateur $\Delta$, ou Laplacien, admet diverses expressions selon le système de coordonnées et les symétries simplificatrices du problème traité. Dans l'étude du refroidissement du cylindre homogène, seule la coordonnée radiale $\rho$ intervient, et l'on peut montrer que le Laplacien s'exprime par

$$
\Delta u=\frac{\partial^{2} u}{\partial \rho^{2}}+\frac{1}{\rho} \frac{\partial u}{\partial \rho}
$$

Quitte à renommer $\rho$ en $x$ pour mieux pouvoir comparer nos écritures dans les différents cas, la température $u(x, t)$ est donnée par les équations (1), (2) et (3)

$$
\begin{aligned}
& \text { (1) } \frac{\partial \mathrm{u}}{\partial \mathrm{t}}=\frac{\partial^{2} \mathrm{u}}{\partial \mathrm{x}^{2}}+\frac{1}{\mathrm{x}} \frac{\partial \mathrm{u}}{\partial \mathrm{x}} \\
& \text { (2) } \mathrm{u}(0, \mathrm{t}) \text { bornée; } \mathrm{u}(1, \mathrm{t})=0 \\
& \text { (3) } \mathrm{u}(\mathrm{x}, 0)=\mathrm{f}(\mathrm{x})
\end{aligned}
$$

On déroule la même technique ; (1) devient

$$
\mathrm{V}(\mathrm{x}) \cdot \mathrm{W}^{\prime}(\mathrm{t})=\left[\mathrm{V}^{\prime \prime}(\mathrm{x})+\frac{1}{\mathrm{x}} \mathrm{V}^{\prime}(\mathrm{x})\right] \cdot \mathrm{W}(\mathrm{t})
$$


et l'on arrive - avec Fourier ! - aux équations

(A) $\quad \mathrm{xV}^{\prime \prime}(\mathrm{x})+\mathrm{V}^{\prime}(\mathrm{x})+\mathrm{rxV}(\mathrm{x})=0$

$$
\text { (A') } \quad \mathrm{W}^{\prime}(\mathrm{t})=-\mathrm{rW}(\mathrm{t})
$$

(B) $\mathrm{V}$ bornée en $0 ; \mathrm{V}(1)=0$

La seule différence, c'est que désormais, l'équation (A) est un peu plus difficile à résoudre. On se ramène au cas $r=1$ par un changement de variable $\xi=\sqrt{\mathrm{r} x}$ [ou $r=-1$, dont on vérifie encore qu'il n'a pas de solution non triviale], et l'on reconnaît alors l'équation de Bessel, dont on sait classiquement rechercher les solutions développables en série entière, pour les lecteurs qui maîtrisent cette technique (que les autres se disent seulement que I'on obtiendra de nouvelles fonctions usuelles que I'on sait étudier et tracer, au même titre que In ou exp). On montre que toutes les solutions bornées en 0 sont proportionnelles à celle, notée $J_{0}$ pour qui $\mathrm{J}_{0}(0)=1$, à savoir

$$
\mathrm{J}_{0}(\xi)=\sum_{\mathrm{k}=0}^{\infty} \frac{(-1)^{\mathrm{k}}}{(\mathrm{k} !)^{2}}\left(\frac{\xi^{2}}{2}\right)^{\mathrm{k}}
$$

Les solutions bornées en 0 de $(A)$ s'expriment alors par

$$
\mathrm{V}(\mathrm{x})=\alpha \mathrm{J}_{0}(\sqrt{\mathrm{r}} \mathrm{x})
$$

mais pour des $r$ racines de l'équation, issue de la condition en $1, V(1)=0$

$$
\text { (C) } \mathrm{J}_{0}(\sqrt{\mathrm{r}})=0
$$

Tout ce travail a été effectué par Fourier, qui, incidemment, a donc rencontré la fonction de Bessel... dix ans avant Bessel (1817) ! 


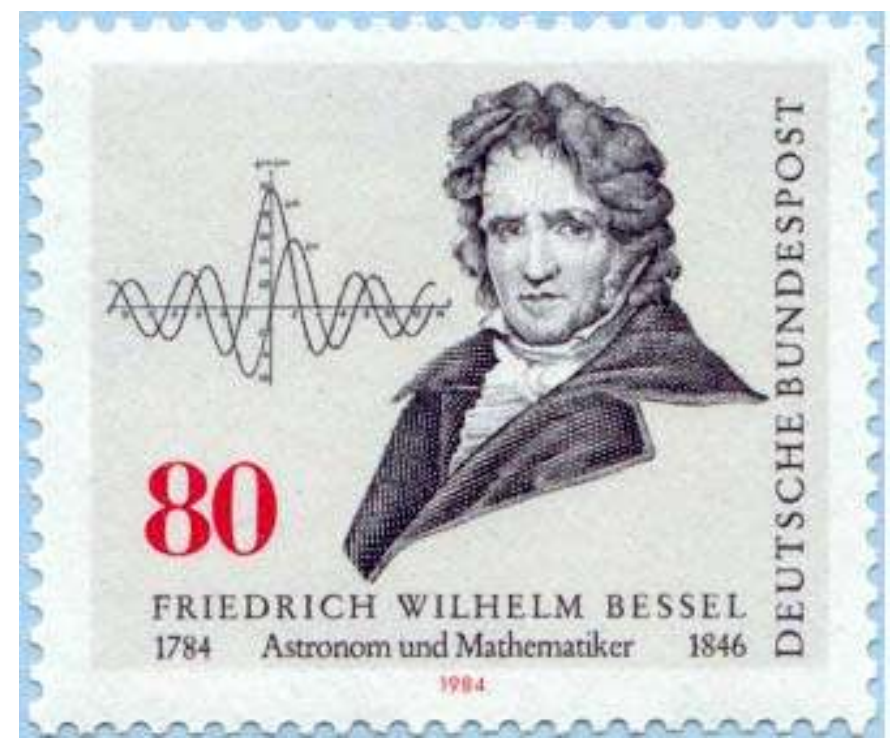

Fiqure 2 : Timbre allemand émis en 1984 à l'occasion du bicentenaire de la naissance de Wilhelm Bessel (1784-1846). À gauche, on reconnaît les deux premières fonctions de Bessel, $J_{O}$ (paire) et $J_{1}$ (impaire). Image J. Miller, site Images of Mathematicians on Postage Stamps.

Sturm et LIOUVille: le temps de la generalisation, la position du PROBLEME

Fourier avait ainsi constitué une collection de cas, selon la géométrie du domaine. Considérer des matériaux non homogènes rend les calculs plus volumineux, mais on obtient les mêmes groupes d'équations, ayant les mêmes significations. Liouville, qui en cela ne fait encore que suivre Sturm, est donc conduit à examiner les équations (1), (2) et (3) traduisant respectivement l'évolution, les conditions aux limites ( $h$ et $H$ sont des constantes), et la condition initiale

$$
\begin{gathered}
g(x) \frac{\partial u}{\partial t}=\frac{\partial}{\partial x}\left[k(x) \frac{\partial u}{\partial x}\right]-I(x) u(x) \\
\frac{\partial u}{\partial x}(a, 0)-h u(a, 0)=0 \\
\frac{\partial u}{\partial x}(b, 0)+H u(b, 0)=0 \\
u(x, 0)=f(x)
\end{gathered}
$$

On conserve dans toute cette présentation, afin de faciliter la lecture du texte 
historique et la mise en correspondance des exemples précédents, les notations et la numérotation adoptées par Liouville. Nous ne ferons qu'une entorse à cette règle, en nommant $a$, au lieu de $\mathrm{x}$ (droit) et $b$, au lieu de $\mathrm{X}$ (droit, majuscule) les bornes de l'intervalle décrit par la variable ...x (italique) dans l'article de Liouville. L'exemple 1 offre un cas de l'extension mentionnée aux valeurs infinies pour $h$ et $H$ : diviser par $h$ ou $H$ efface le premier terme, puisque tout se passe comme si son coefficient était nul. La séparation des variables conduit facilement, de manière similaire, à

$$
\begin{gathered}
{\left[\mathrm{k}(\mathrm{x}) \mathrm{V}^{\prime}(\mathrm{x})\right]^{\prime}+[\mathrm{rg}(\mathrm{x})-\mathrm{I}(\mathrm{x})] \mathrm{V}(\mathrm{x})=0} \\
\mathrm{~V}^{\prime}(\mathrm{a})-\mathrm{h} \mathrm{V}(\mathrm{a})=0 \\
\mathrm{~V}^{\prime}(\mathrm{b})+\mathrm{H} \mathrm{V}(\mathrm{b})=0 \\
\mathrm{u}(\mathrm{x}, 0)=\mathrm{f}(\mathrm{x})
\end{gathered}
$$

et Liouville ajoute que la réalisation de (B) impose, pour obtenir des solutions non identiquement nulles, de prendre $r$ parmi les racines d'une équation qu'il note (p.256):

(C) $\varpi(r)=0$

et qu'il qualifie de transcendante, car elle a, dans tous les exemples rencontrés, une infinité dénombrable de racines $r_{n}$ : il ne peut donc s'agir d'une équation polynomiale.

Un lecteur perspicace ne manquera pas de constater que nous ne nous sommes jamais occupés, jusqu'ici, de la condition ( 3 ) ; c'est d'elle que provient la question qui fonde l'article de Liouville. Mais d'abord, voyons ce qu'il en était dans le cas le plus simple, celui de Fourier, dans l'exemple 1. La série se traduit par

$$
f(x)=u(x, 0)=\sum_{n=1}^{\infty} \beta_{n} V_{n}(x) W_{n}(0)=\sum_{n=1}^{\infty} \beta_{n} \sin (\pi n x)
$$

C'est sous cette forme que Fourier avait rencontré la question du 
développement qui porte son nom ! En d'autres termes : $f$ étant donnée, trouver les coefficients $\beta_{n}$ convenables, et obtenir ainsi une série solution des conditions (1), (2) et (3). Le but que s'assigne Liouville, à la fin du §I de son texte, en est l'exacte généralisation :

On peut voir, dans l'ouvrage de M. Poisson sur la chaleur, comment on est porté, par la marche même du calcul, à admettre la possibilité de ce développement pour une fonction quelconque $f(x)$; mais jusqu'à ce jour il a paru difficile d'établir cette possibilité directement et d'une manière rigoureuse. Je me propose de donner ici une méthode très simple pour $y$ parvenir.

Dans l'exemple 2, il s'agit de tenter un développement, toujours en série de sinus, mais de pulsations plus irrégulières; et dans l'exemple 3 , de rechercher un développement en série de Bessel

$$
f(x)=u(x, 0)=\sum_{n=1}^{\infty} \beta_{n} V_{n}(x) W_{n}(0)=\sum_{n=1}^{\infty} \beta_{n} J_{0}\left(\omega_{n} x\right)
$$

On mesure ainsi tout à la fois l'ambition du programme et la fécondité de l'approche par généralisation, qui est une première dans ce domaine.

\section{Les Predecesseurs : Fouri er, PoIsson, Sturm et les Autres...}

De Fourier, les exemples introductifs nous ont amené à parler abondamment. La convergence de la série développant $f$, point mathématiquement si sensible, ne l'intéressait guère puisqu'elle n'était qu'un auxiliaire de calcul des coefficients $\beta_{n}$ qui, pour $\mathrm{t}>0$, pondéraient des exponentielles dont la forte décroissance garantissait «moralement » la convergence dans tous les cas issus de la physique : aurait-on obtenu pour tout $n: \beta_{n}=1$, l'écriture de $f$ en série n'aurait eu aucun sens, mais cela n'aurait pas empêché la série de $u$ de converger et de vérifier (1). En étudiant le problème pour lui-même, dégagé et de la source physique (la chaleur) et de la source mathématique (l'équation aux dérivées partielles), Liouville s'engage sur un terrain où l'intérêt le dispute au risque :

Cette fonction $V$, comme on vient de le voir, se présente utilement dans la 
théorie de la chaleur; mais nous la considérons ici en elle-même, abstraction faite de son usage en physique mathématique.

La première étude sérieuse de convergence est tout fraîche (Dirichlet 1829) Iorsque Liouville aborde ces questions (dès 1830), et elle est d'une rigueur parfaite... mais elle requiert des hypothèses un peu trop contraignantes au goût même de son auteur.

À Poisson, Liouville doit la question du développement - et l'insuffisance de sa justification. Liouville va également exploiter, dans son calcul, des relations d'orthogonalité que Poisson est le premier à faire apparaître

$$
\int_{a}^{b} V_{j}(x) V_{k}(x) g(x) d x=0
$$

et même à étendre audacieusement à des fonctions à valeurs complexes, afin de prouver la réalité de tous les $r_{n}$ rencontrés.

Séparer les contributions de Sturm et Liouville semble a priori plus délicat, tant l'étude est désormais connue sous l'appellation Problème de Sturm-Liouville. Si Sturm a précédé Liouville - les dates de leurs communications respectives à l'Académie en font foi - un article à quatre mains est écrit pour le deuxième numéro du Journal de Liouville, en 1837, et la parution des articles de Sturm doit beaucoup à l'insistance de Liouville; ce dernier le rappelait sur la tombe de son ami, lors de ses funérailles, en 1855 :

Deux beaux Mémoires sur la discussion des équations différentielles et à différences partielles, propres aux grands problèmes de la physique mathématique, ont été du moins publiés en entier, grâce à mon insistance. La postérité impartiale les placera à côté des plus beaux Mémoires de Lagrange.

Toutefois, si l'on classe les divers aspects des problèmes aux limites en trois rubriques principales:

1. Étude de la famille des valeurs $r_{n}$ de $r$ donnant des solutions non triviales ;

2. étude qualitative des solutions $v_{n}$ associées ;

3. développement d'une fonction $f$ donnée en série des $v_{n}$, on peut dire que les travaux de Sturm concernent les deux premiers aspects, ceux de Liouville le troisième, comme il l'a annoncé lui-même au début de son texte. 
Premier exemple de l'étude qualitative approfondie des solutions d'une équation différentielle du second ordre, les mémoires de Sturm contiennent notamment les célèbres théorèmes d'oscillation et de comparaison, grâce auxquels les points où une solution s'annule peuvent être organisés en une suite croissante : Sturm établit leur entrelacement avec les zéros d'une solution d'une équation à coefficients constants, donc aisément résoluble. Ce qui est essentiel dans un cas comme notre exemple 3, où ces zéros participent directement à la définition des $r_{n}$. Dans ce cas, la théorie de Sturm montre l'oscillation de la fonction, son amortissement, et elle prouve l'existence d'une infinité de racines pour l'équation (C) .

\section{OUVI LLE ET LE DEVELOPPEMENT EN SERI E DE FONCTIONS ORTHOGONALES}

Un calcul purement formel - c'est à dire, fait de manipulations algébriques sans justification de convergence ni d'échange d'intégrale et de sommation, « à la Euler », pourrait-on dire - d'ailleurs, celui-ci l'avait effectué sur une série de Fourier particulière - suggère l'analyse suivante :

si $f(x)=\sum_{n=1}^{\infty} \beta_{n} V_{n}(x)$, multipliant par $g(x) V_{p}(x)$ et intégrant de a à $b$, on aura

$$
\int_{a}^{b} f(x) V_{p}(x) g(x) d x=\sum_{n=1}^{\infty} \beta_{n} \int_{a}^{b} V_{n}(x) V_{p}(x) g(x) d x
$$

dont un seul terme subsiste grâce aux relations d'orthogonalité du type Poisson

$$
\int_{a}^{b} f(x) V_{p}(x) g(x) d x=\beta_{p} \int_{a}^{b} V_{p}(x)^{2} g(x) d x
$$




\section{Les relations d'orthogonalité de Poisson}

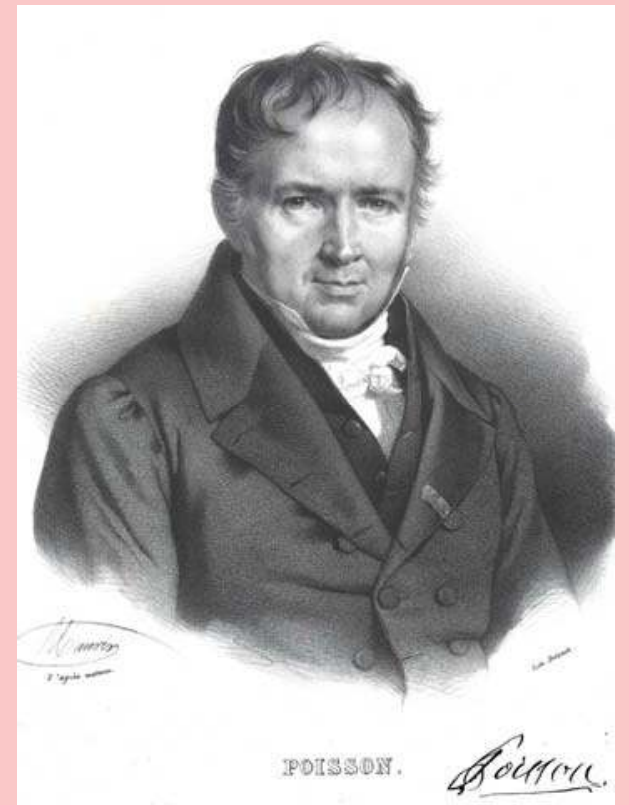

Figure 3 : Siméon- Denis Poisson (1781-1840)

D'où viennent de telles relations ? De deux choses :

1. de la forme très particulière, dite autoadjointe, de l'équation (A) une équation qui n'est pas sous cette forme nécessite un petit travail de préparation pour s'y ramener;

2. des conditions aux limites (B).

En écrivant, en effet, que $v_{n}$ est solution de (A) pour la valeur $r_{n}$ sous la forme

$$
r_{n} g V_{n}=I V_{n}-\left[k V_{n}^{\prime}\right]^{\prime}
$$

on multiplie par $V_{p}$ et on intègre de $a$ à $b$

$$
r_{n} \int_{a}^{b} V_{n} V_{p} g d x=\int_{a}^{b} I V_{n} V_{p} d x-\int_{a}^{b}\left[k V_{n}^{\prime}\right] V_{p} d x
$$

puis, par parties:

$$
\begin{aligned}
& r_{n} \int_{a}^{b} V_{n} V_{p} g d x=\int_{a}^{b} I V_{n} V_{p} d x+\int_{a}^{b} k V_{n}^{\prime} V_{p}^{\prime} d x-\left[k V_{n}^{\prime} V_{p}\right](b)+\left[k V_{n}^{\prime} V_{p}\right](a) \\
& r_{n} \int_{a}^{b} V_{n} V_{p} g d x=\int_{a}^{b} I V_{n} V_{p} d x+\int_{a}^{b} k V_{n}^{\prime} V_{p}^{\prime} d x+\left[k H V_{n} V_{p}\right](b)-\left[k h V_{n} V_{p}\right](a)
\end{aligned}
$$

grâce aux conditions (B ); mais comme l'expression est symétrique en $n$ et p

$$
r_{n} \int_{a}^{b} V_{n} V_{p} g d x=r_{p} \int_{a}^{b} V_{n} V_{p} g d x
$$


On n'intègre pas des sommes infinies comme des sommes ordinaires, quoique Cauchy, apôtre de la rigueur s'il en est, le pratique encore couramment. Les premières justifications solides viendront avec la convergence uniforme de Weierstrass (1861) : ni Fourier, ni Poisson, ni Dirichlet ne disposent d'un tel outil. Liouville n'est pas mieux loti, mais propose un autre angle d'attaque :

1. après tout, peu importe la rigueur de l'analyse, si elle nous permet de deviner la bonne formule ;

2. partons donc de la série selon la formule suggérée et appliquons toute notre rigueur à la synthèse: il suffit de sommer la série... et de trouver $f$.

Il insiste sur cette approche, au point de le dire deux fois, à la fin du §I d'abord, puis à celle du §II :

Sans rien supposer a priori sur l'origine de cette série ni sur sa nature, j'en cherche la valeur, et je trouve que cette valeur est $f(x)$.

Notre but, dans ce mémoire, est de trouver directement et par un procédé rigoureux la somme de la série $\sum\left\{\frac{\mathrm{V} \int_{\mathrm{a}}^{\mathrm{b}} \mathrm{gVf}(\mathrm{x}) \mathrm{dx}}{\int_{\mathrm{a}}^{\mathrm{b}} \mathrm{gV}^{2} \mathrm{dx}}\right\}$, dans laquelle le signe $\Sigma$ s'étend à toutes les valeurs de $r$ qui satisfont l'équation (C). Quelle que soit la fonction $f(x)$, nous montrerons que la série en question a précisément pour somme $f(x)$.

\section{DES NOTATIONS MODERNES : UN SOULAGEMENT !}

Poser les notations d'un produit scalaire sur les espaces de fonctions est évidemment un anachronisme, mais quel confort le lecteur peut en retirer aujourd'hui ! Quelle satisfaction aussi de voir combien les progrès de « l'équipement technique » des mathématiques rendent accessibles et manipulables par un étudiant de premier cycle des calculs que seuls suivaient des chercheurs un siècle et demi auparavant! Notons donc 


$$
\langle\varphi, \psi\rangle=\int_{\mathrm{a}}^{\mathrm{b}} \varphi(\mathrm{x}) \psi(\mathrm{x}) \mathrm{g}(\mathrm{x}) \mathrm{dx}
$$

Les relations que nous avons qualifiées «d'orthogonalité » méritent cette dénomination

$$
\left\langle V_{j}, V_{k}\right\rangle=\int_{a}^{b} V_{j}(x) V_{k}(x) g(x) d x=0
$$

et la série dont parle Liouville s'allège en

$$
\sum_{\mathrm{k}=1}^{\infty} \frac{\left\langle\mathrm{V}_{\mathrm{k}}, \mathrm{f}\right\rangle}{\left\langle\mathrm{V}_{\mathrm{k}}, \mathrm{V}_{\mathrm{k}}\right\rangle} \mathrm{V}_{\mathrm{k}}(\mathrm{x})=\sum_{\mathrm{k}=1}^{\infty} \mathrm{c}_{\mathrm{k}}(\mathrm{f}) \mathrm{V}_{\mathrm{k}}(\mathrm{x})
$$

OU L'OPERATI ON REUSSIT... AU PRIX DE DEUX LEMMES (ET D'UN « PETIT » ABUS) !

Pour ne pas nous égarer dans un intermède technique, sautons jusqu'au $\S \vee$ et regardons Liouville opérer. Avec sagesse, il donne un nom différent à la série $F(x)$; il s'agit donc de montrer que $F(x)=f(x)$.

Le calcul effectué est alors le même que celui de l'analyse, mais en remplaçant $f$ par $F$, et il n'est évidemment pas mieux justifié !

Je multiplie les deux membres par $\mathrm{g}(\mathrm{x}) \mathrm{V}_{\mathrm{m}}(\mathrm{x}) \mathrm{dx}$ et j'intègre ensuite par rapport à $x$ en prenant a et $b$ pour les limites de l'intégrale.

explique-t-il. Bref, il écrit, toujours form ellem ent, en exploitant l'orthogonalité

$$
\begin{gathered}
\left\langle\mathrm{V}_{\mathrm{m}}, \mathrm{F}\right\rangle=\left\langle\mathrm{V}_{\mathrm{m}}, \sum_{\mathrm{k}=1}^{\infty} \mathrm{c}_{\mathrm{k}}(\mathrm{f}) \mathrm{V}_{\mathrm{k}}\right\rangle \\
\left\langle\mathrm{V}_{\mathrm{m}}, \mathrm{F}\right\rangle=\sum_{\mathrm{k}=1}^{\infty} \mathrm{c}_{\mathrm{k}}(\mathrm{f})\left\langle\mathrm{V}_{\mathrm{m}}, \mathrm{V}_{\mathrm{k}}\right\rangle=\mathrm{c}_{\mathrm{m}}(\mathrm{f})\left\langle\mathrm{V}_{\mathrm{m}}, \mathrm{V}_{\mathrm{m}}\right\rangle
\end{gathered}
$$

On a donc, pour tout $m, c_{\mathrm{m}}(\mathrm{F})=\mathrm{c}_{\mathrm{m}}(\mathrm{f}) \mathrm{ou}$, en termes de produit scalaire

$$
\forall \mathrm{m},\left\langle\mathrm{V}_{\mathrm{m}}, \mathrm{F}-\mathrm{f}\right\rangle=0
$$

De là à conclure $F=f$, il n'y a qu'un pas... et c'est à ce travail que sont dévolus les lemmes. 


\section{LES LEMMES OU L'ESPOI R DERAI SONNABLE}

Dans un espace géométrique de dimension 3, de base orthonormée donnée ( $\mathbf{i}$, $\mathbf{j}, \mathbf{k}$ ), un vecteur orthogonal aux trois vecteurs de la base est nul, c'est facile à prouver. Mais déjà, qu'on en oublie un, et l'on n'arrivera à rien : un vecteur orthogonal à $\mathbf{i}$ et $\mathbf{j}$... a parfaitement le droit d'être colinéaire à $\mathbf{k}$ !

Il en irait de même dans tout espace de dimension finie : par exemple l'espace de fonctions combinaisons linéaires de $\sin x, \sin 2 x, \ldots, \sin N x$, avec $N$ fixé. Ou celui des combinaisons linéaires de $v_{1}, V_{2}, \ldots, v_{N}$. Aussi grand soit $N$, pourvu qu'il soit fixé.

Mais qu'en est-il lorsqu'on s'intéresse, et c'est toujours le cas dans le problème de Sturm-Liouville, à une infinité de vecteurs orthogonaux entre eux ? En a-t-on «assez » pour conclure de même, ou ne risque-t-il pas d'en manquer, comme dans l'expérience naïve où nous n'avions pris que deux vecteurs sur trois ? Mais hélas, de façon bien moins apparente...

Et de fait, la question ne reçoit pas toujours la même réponse. Cela a motivé la distinction des «bons » systèmes, ceux qui généralisent à la dimension infinie les «bonnes » propriétés d'une base orthonormale en dimension finie : on les appelle bases hilbertiennes, en hommage à l'étude menée par Hilbert à partir de 1904. Une des façons de les introduire est justement de les définir comme des familles totales, c'est-à-dire vérifiant l'axiome

$$
\forall \mathrm{m},\left\langle\mathrm{V}_{\mathrm{m}}, \varphi\right\rangle=0 \Rightarrow \varphi=0
$$

Le système des $(\cos n ., \sin n$.$) de l'analyse de Fourier est un exemple de$ système total. Mais la preuve n'en est pas immédiate; elle requiert des théorèmes auxiliaires importants. De façon générale, prouver la totalité d'un système n'est jamais une petite tâche anodine... c'est dire que Liouville s'attaquait - pour l'époque ! - à plus fort que lui, et que sa tentative ne pouvait qu'être vouée à l'échec. Mais, de manière bien plus positive, on doit considérer que cette lacune a beaucoup fait pour la position claire de cette question et le lancement des espaces de Hilbert.

Le lemme 2 de Liouville (§IV) affirme exactement la totalité de la famille des $V_{m}$. $\varphi$ désignant toujours la fonction dont il s'agit d'établir la nullité, supposons 
d'abord que $\varphi$ ne change de signe qu'en un nombre fini de points, $a_{1}, a_{2}, \ldots, a_{m-1}$ (Liouville n'utilise pas l'indexation, les dénommant seulement $a, b, c, \& c . .$. ). Il construit, grâce au lemme 1 , une combinaison linéaire $\Psi$ des $V_{k}$ possédant les mêmes zéros exactement, de même signe que $\varphi$. De la sorte,

$$
\langle\varphi, \psi\rangle=\int_{a}^{b} \varphi(x) \psi(x) g(x) d x
$$

est à la fois nul et strictement positif, d'où la contradiction souhaitée.

Le lemme $1(\S I V)$ n'est qu'une construction progressive de $\Psi$ à partir des $V_{m}$ : annulation et changement de signe en $a, b$, puis en $a, b, c$, et ainsi de suite. C'est sans mystère.

Le «seul » problème, c'est que nous avons traité le cas d'un nombre fini de changements de signe, pas d'une infinité. Or, une fonction, même très régulière, peut posséder une infinité de zéros : l'exemple de $x \longrightarrow x \sin \frac{1}{x}$ est bien connu pour être continu, et $x \longrightarrow x^{3} \cdot \sin \frac{1}{x}$ de classe $C^{1}$. Des notes ultérieures de Liouville (1840) montrent qu'il a dû concéder cette imperfection, pointée sans doute par Dirichlet.

\section{ÉTAPE SUI VANTE : LES ESPACES DE HI LBERT...}

Au tournant du XXe siècle, un progrès notoire, réalisé par Hilbert (entre 1904 et 1910) et Schmidt (1907), a été d'abandonner le point de vue ponctuel pour les écritures du type

$$
\sum_{k=1}^{\infty} \frac{\left\langle V_{k}, f\right\rangle}{\left\langle V_{k}, V_{k}\right\rangle} V_{k}=\sum_{k=1}^{\infty} c_{k}(f) V_{k}
$$

et de proposer pour de telles séries - à commencer, bien entendu, par les séries de Fourier - un sens global, l'écriture étant définie, via la norme associée au produit scalaire, par 


$$
F=\sum_{k=1}^{\infty} c_{k}(f) V_{k} \Leftrightarrow \lim _{N \rightarrow \infty}\left\|F-\sum_{k=1}^{N} c_{k}(f) V_{k}\right\|=0
$$

Les fonctions deviennent les vecteurs d'un espace de Hilbert qui s'avère être le cadre naturel pour une étude à hypothèses les moins contraignantes possibles. Un théorème, dit de Hilbert-Schmidt, permet alors de récupérer, dans certains cas (dont celui du problème de Sturm-Liouville) la convergence ponctuelle, voire uniforme. L'étude spectrale, elle aussi, s'allège remarquablement: la forme autoadjointe de l'équation $(A)$ se traduit par le caractère autoadjoint de l'opérateur $\mathrm{D}$ défini par $\varphi \rightarrow \mathrm{D} \varphi=\frac{1}{\mathrm{~g}}\left[\left(\mathrm{k} \varphi^{\prime}\right)^{\prime}-\mathrm{l} \varphi\right]$ :

$$
\langle\mathrm{D} \varphi, \psi\rangle=\langle\varphi, \mathrm{D} \psi\rangle
$$

et l'orthogonalité des fonctions propres apparaît comme une conséquence générale de cette propriété.

Si cette théorie fait partie, aujourd'hui, du bagage de tout étudiant en mathématiques ou futur ingénieur, si ces espaces sont le bon cadre pour la théorie du signal, il est juste de rappeler ce que son essor doit à l'imperfection des écrits de Liouville, comme à l'urgente nécessité des applications pratiques.

\section{Le « liouvillien » en physique quantique}

Le travail d'analyse qu'a mené Liouville autour de l'équation de la chaleur de Fourier est aussi utilisé dans sa plus grande généralité en physique quantique, pour certaines résolutions de l'équation de Schrödinger. Comme le rappelle Claude Cohen-Tannoudji dans son cours au Collège de France ${ }^{2}$, I'espace des opérateurs agissant sur l'espace $\mathcal{E}$ des états d'un système quantique est appelé « espace de Liouville » et noté $\mathcal{L}$.

Il est défini, dans cet espace d'opérateurs, un opérateur particulier, le Liouvillien (ou «opérateur de Liouville») : à partir de l'opérateur hamiltonien $\mathrm{H}$ agissant dans l'espace des états $\mathcal{E}$, on définit I'opérateur de Liouville $L$ agissant dans I'espace $\mathcal{L}$.

2. http://www.phys.ens.fr/cours/college-de-france/1975-76/1975-76.htm, Année universitaire 1975-1976, «Emission spontanée, relaxation et équation pilote », $8^{\circ}$ leçon. 


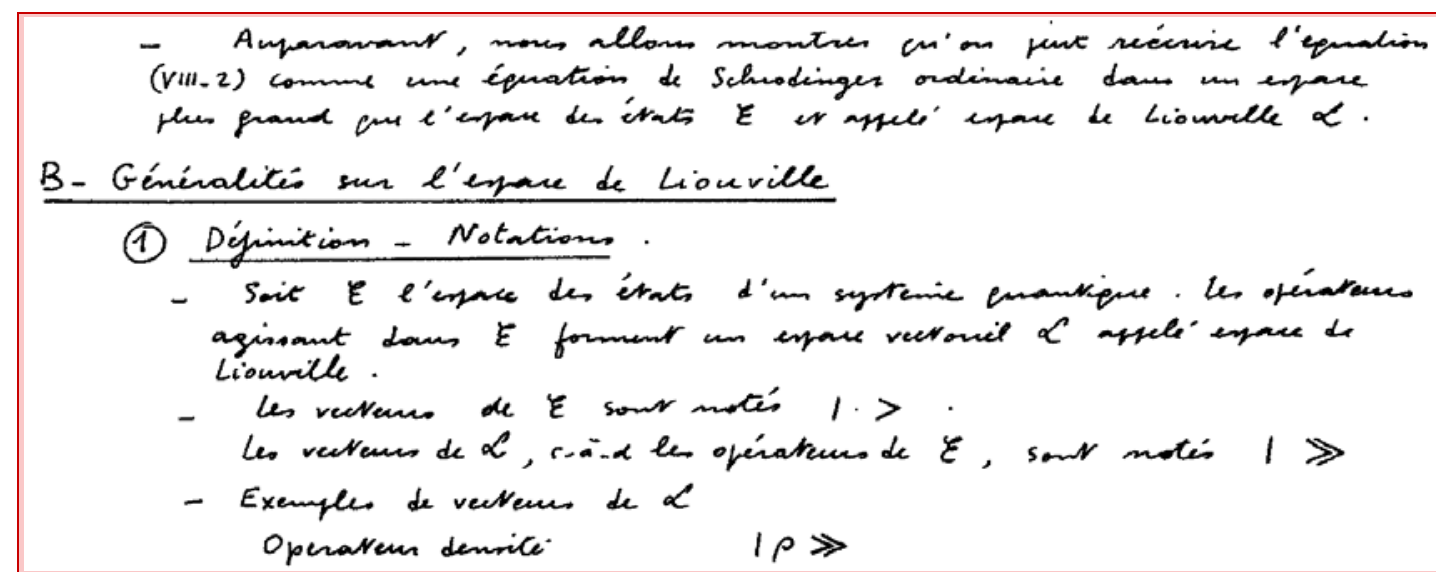

L'opérateur de Liouville $L$ permet d'écrire différemment certaines équations fondamentales de la physique quantique - comme celle de Schrödinger déjà citée ou celle des observables de Heisenberg - et d'aider à leur résolution :

(ii) Intéiet de L.

- L'équation d'évoluction de $\rho$, vill-2, pent ítre récuire dones $\alpha$, compte teme de $(v i 11-18)$, sour la forme

$$
i \hbar \frac{d}{d t}|\rho \gg=L| \rho \gg
$$

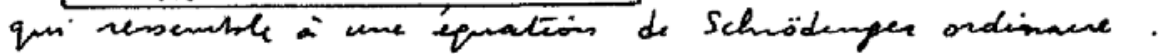

- De minne l'epuation d'érolutioin d'une obsemaste A

$i \hbar \frac{d}{d c} A=[A, H]$

$(v 111-20)$

jent se réecure

$i \hbar \frac{d}{d t}|A \geqslant=-L| A \gg$

$(v i 11-21)$

On constate ainsi la puissance des outils créés par Liouville en analyse, utilisés en physique mathématique : les apports de Liouville à la résolution de l'équation différentielle de la chaleur sont de nos jours utilisés pour la résolution des équations de la physique quantique.

\section{CONCLUSION}

La portée d'un article de mathématiques ne tient pas qu'à la rigueur de ses preuves; elle se juge aussi à la fécondité des problèmes soulevés, voire à la postérité qu'il suscite sous la forme de démonstrations complémentaires ou de nouveaux concepts. Dans la théorie des séries de Fourier, le mémoire de Fourier, malgré ou à cause de ses insuffisances, ouvre un champ de travail pour deux cents ans de mathématiques : il est du deuxième type évoqué, tandis que celui de Dirichlet marque un jalon du premier type, y compris dans l'histoire des 
mathématiques.

Le mémoire de Liouville, et plus généralement l'ensemble des articles que Sturm et Liouville consacrent à ce thème, procèdent des deux registres. Liouville écrit les premières pages du grand livre des développements en séries de fonctions orthogonales, dont la récente théorie des ondelettes (1980) est le dernier - et spectaculaire - avatar. Il prépare le tournant de l'analyse hilbertienne et stimule l'étude des familles orthogonales totales.

Ce même problème a inspiré à Sturm les premières études qualitatives des équations différentielles; valeurs et fonctions propres ont fait à cette occasion une entrée fracassante dans le monde des mathématiques,.. et, de façon naturelle, en dimension infinie ! On s'en voudrait de l'oublier, quand tous nos enseignements (dans un louable souci de progressivité, reconnaissons-le...) abordent systématiquement ces notions dans le cadre de la dimension finie. L'histoire n'a donc pas choisi de commencer par les problèmes les plus simples...

Enfin, n'oublions pas de verser une dernière pièce, et non la moindre, à la rubrique « rigueur »: c'est dans son second mémoire que Liouville a introduit la méthode des approximations successives pour une équation différentielle du second ordre, afin de prouver l'existence et unicité d'une solution au problème avec conditions initiales, avant la généralisation qu'en donnera Émile Picard en 1890. C'est la première démarche de ce genre pour une équation du second ordre, Cauchy s'étant, quant à lui, occupé de celles du premier ordre.

Il ne sera donc pas illégitime d'appliquer à cet opus de Liouville le commentaire que Jesper Lützen fait sur l'article de Sturm: «Peu d'autres articles, en mathématiques, peuvent rivaliser avec celui-ci en termes de nouveauté du problème, des méthodes et des résultats ». Et d'ajouter, pour fêter dignement le bicentenaire de sa naissance : merci à vous, M. Liouville, d'avoir donné tant de beau travail à faire à tant de grands mathématiciens!

(juin 2010)

(cet article a été publié une première fois dans le Bulletin de la SABIX, $n^{\circ} 45$, janvier 2010, consacré à Liouville ; l'encadré hors texte pages 18-19, lui aussi publié dans ce Bulletin, est d'Alexandre Moatti) 